\title{
O PAPEL DO TAMANHO DAS PARTICULAS DE AGREGADO DE QUARTZO NA DURABILIDADE DE ARGAMASSAS GEOPOLIMÉRICAS
}

\author{
LUCIANO SOUSA BATISTA, LUCAS \\ Graduação em Engenharia Civil \\ Instituto de Tecnologia da Universidade Federal do \\ Pará, FEC - GAEMA - ACGEO \\ Pará, Brasil \\ lucas.lsb@outlook.com
}

\author{
SOUZA PICANÇO, MARCELO \\ Professor Doutor \\ Instituto de Tecnologia da Universidade Federal do \\ Pará, PPGEC - FEC - GAEMA - ACGEO \\ Pará, Brasil \\ marcelosp@ufpa.br
}

GOMES-PIMENTEL, MAURÍLIO

Doutorando em Engenharia Civil

Instituto de Tecnologia da Universidade Federal do

Pará, PPGEC - FEC - GAEMA - ACGEO

Pará, Brasil

mauriliogpimentel@gmail.com

\author{
GOMES GALIZA-JUNIOR, JONAS \\ Graduação em Engenharia Civil \\ Instituto de Tecnologia da Universidade Federal do \\ Pará, FEC - GAEMA - ACGEO \\ Pará, Brasil \\ jonasggjunior@gmail.com
}

\author{
FRANCO CORRÊA DOS SANTOS, SIDNEY \\ Graduação em Engenharia Civil \\ Instituto de Tecnologia da Universidade Federal do \\ Pará, FEC - GAEMA - ACGEO \\ Pará, Brasil \\ sidneyf.correa@gmail.com
}

\section{RESUMO}

O Império Romano Ocidental desmoronou há mais de mil anos, mas suas estruturas de concreto ainda estão de pé e são conhecidas por sua beleza e especialmente sua durabilidade quando comparada as estruturas atuais. A baixa vida útil de muitas estruturas modernas tem ressaltado a importância de estudos relacionados a durabilidade, especialmente de materiais novos como o geopolímero, um ligante formado pela ativação alcalina de um aluminossilicato, um material promissor para produção de compósitos, mas pouco se sabe sobre sua durabilidade e os efeitos de sua interação com as partículas de agregado de quartzo. Aqui, foi investigado o efeito do tamanho das partículas de quartzo na durabilidade da argamassa geopolimérica. Os resultados revelam que a superfície específica e o empacotamento dos agregados como principais controladores da durabilidade do geopolímero, e contribuem para uma teoria de dissolução da sílica do agregado, fortalecendo a zona de transição da argamassa geopolimérica. Portanto, um ajuste adequado da granulometria do agregado, implica em um maior controle da durabilidade, e consequentemente uma diminuição de manifestações patológicas nos compósitos geopoliméricos.

Palavras chave: Argamassa ecológica, patologia, trincas, álcali ativado, vida útil.

\begin{abstract}
The Western Roman Empire collapsed more than a thousand years ago, but its concrete structures are still standing and are known for their beauty and especially their durability when compared to current structures. The low service life of many modern structures has highlighted the importance of studies related to durability, especially of new materials such as geopolymer, a binder formed by the alkaline activation of an aluminosilicate, a promising material for the production of composites, but little is known about its durability and the effects of its interaction with the aggregate particles. Here, the effects of quartz particle size on the durability of the geopolymeric mortar were investigated, the results reveal the specific surface and packaging of the aggregates as the main controllers of the durability of the geopolymer, and contribute to a theory of dissolution of the aggregate silica, strengthening the transition zone of the geopolymeric mortar. Therefore, an adequate adjustment of the aggregate granulometry, implies a greater control of durability, and consequently a decrease in pathological manifestations in geopolymeric composites.
\end{abstract}

Keywords: geopolymer, durability, transition zone, quartz, mortar. 


\section{INTRODUÇÃO}

Há cerca de 2000 anos, Plinio, o Velho, mencionou as estruturas romanas feitas pelo engenheiro Vitrúvio, como sendo inexpugnáveis pelas ondas e cada dia mais fortes (PLINIO, 77AD), a grande prova de que Plinio estava certo é a durabilidade que mantem essas estruturas de pé até os dias de hoje (DELATTE, 2001), e os estudos atuais sobre seu comportamento, além de recriações dessas argamassas e concretos (BRUNE et al., 2013; JACKSON et al., 2014, 2017). Após o fim do grande império romano, todo esse conhecimento foi perdido e hoje os estudos sobre durabilidade procuram recriar estruturas e materiais tão duráveis ou mais que os romanos.

A durabilidade pode ser definida como a capacidade do material de não perder suas propriedades ao longo do tempo, de modo que na engenharia, a durabilidade também é uma forte influenciadora nos fatores econômicos e sustentáveis, evidenciando a importância da durabilidade em compósitos utilizados na construção (SCHIESSL, 1996). Desta forma, o conceito de durabilidade está fortemente ligado ao de patologia, pois as patologias são uma das principais causas de perda das propriedades do material e consequentemente da diminuição de sua vida útil.

As patologias são promovidas por vários agentes externos, mas são fatores intrínsecos do próprio material que facilitam a ação dos agentes patológicos (SHIN; LANGE, 2004), como as fissuras e a porosidade. Dessa forma, se torna possível e lógico, estudar a durabilidade como uma propriedade também responsável pelo combate das patologias, e através das respostas do material em propriedades que determinam a sua permeabilidade, podemos inferir e discutir sobre sua durabilidade.

O estudo de durabilidade foi amplamente realizado em materiais a base de cimento Portland (HANSEN, 1987; BOIVIN et al., 1999; KLYSZ; BALAYSSAC, 2007; CHEAH; RAMLI, 2012; ZHANG; ZAKARIA; HAMA, 2013; MARUYAMA; SUGIE, 2014), porém, devido ao grande impacto ambiental da produção do cimento Portland, surge um material promissor para substituir o cimento convencional, o geopolímero, um aluminossilicato álcali ativado com propriedades ligantes. Por apresentar ótimas resistências mecânicas e ser resistente a ataques químicos (ZHUANG et al., 2016) e ainda emitir menos carbono em sua produção (DUXSON et al., 2007a), ele se torna uma excelente alternativa aos materiais cimentícios convencionais. Contudo, um dos grandes limitantes do geopolímero na construção civil é a falta de comprovação prática da sua durabilidade, por não existir obras de idade suficiente para caracterizar seus comportamentos a longo prazo, como é feito com o cimento Portland. Por isso é ainda mais importante e significativo para a ciência, utilizar de respostas indiretas, todavia, relacionados com a durabilidade.

Uma das respostas indiretas, é a retração (CHEAH; RAMLI, 2012), ou seja, a diminuição de volume do material devido à perda de água para o ambiente no caso da retração por secagem (HANSEN, 1987), ou devido as reações internas do compósito quando se trata da retração autógena (BOIVIN et al., 1999). Assim, sendo comumente relacionada ao surgimento de trincas e fissuras no material devido a essa variação dimensional, ocasionando diminuição da resistência mecânica e enfraquecendo sua proteção contra agentes externos que podem vir a causar patologias no material.

Os estudos desenvolvidos até agora sobre a retração em materiais geopoliméricos mostram que a retração depende do tipo de cura utilizada (PERERA et al., 2007), bem como das relações molares (KUENZEL et al., 2012), e principalmente do agregado (STEINEROVA, 2011; YANG; ZHU; ZHANG, 2017; HAJIMOHAMMADI; NGO; VONGSVIVUT, 2019). Esses são fatores que também influenciam em materiais a base de cimento Portland, entretanto, a maior parte dos trabalhos concordam que a relação com agregados em geopolímeros é completamente diferente quando comparada ao cimento convencional.

Outra propriedade importante na definição da durabilidade de argamassas e concretos, é a absorção total, pois é uma forma de mensurar o volume de poros conectados em um material e consequentemente sua densidade aparente e medir indiretamente sua resistência. Em argamassas geopoliméricas contribui para determinar a durabilidade em vista que os poros definem a permeabilidade do material tornando-o mais suscetível a entrada de agentes agressivos e consequentemente o surgimento de patologias no material. Mermerdaş et al., 2017, mostra que uma granulometria dos agregados bem distribuída favorece a diminuição da absorção nas argamassas, mas ainda é inconclusivo se essa variação é puramente pela variedade maior da granulometria ou pelo tamanho global das faixas granulométricas utilizadas.

A trabalhabilidade é também uma propriedade determinante na durabilidade, pois ela define a facilidade que o material tem de ser utilizado mantendo a homogeneidade (KOEHLER, 2009) e isso é essencial para evitar a falha em moldagens. E a variação granulométrica costuma influenciar na reologia (CHOI et al., 2014), tornando uma propriedade importante para se estudar quando se tem uma variação nos tamanhos do agregados. 
Desta forma, fica claro que a retração, absorção e outros fatores que determinam a durabilidade dos materiais são intimamente ligados ao combate às patologias e é evidente que a durabilidade é influenciada pelo tamanho das partículas de agregado, porém, não está claro como essa influência ocorre nas propriedades que definem a durabilidade das argamassas geopoliméricas. Assim, este trabalho se propôs a estudar a interferência do tamanho das partículas do agregado de quartzo na retração, absorção total, espalhamento e resistência a compressão, em argamassas geopoliméricas para inferir sobre sua durabilidade.

\section{MATERIAIS E MÉTODOS}

\subsection{Materiais}

Foi utilizado no presente estudo como precursor para a argamassa geopolimérica o metacaulim (MK) disponibilizado comercialmente, e funcionará como a principal fonte de aluminossilicatos para a formação do geopolímero. O MK apresenta massa específica de $2,33 \mathrm{~g} / \mathrm{cm}^{3}$ e a composição química é mostrada na Tabela 1, obtida por meio da Fluorescência de Raio X (FRX) do material. Os ativadores alcalinos utilizados foram: o hidróxido de sódio ( $\mathrm{NaOH}, 98 \%$ de pureza) em escama, e o silicato de sódio $\left(\mathrm{Na}_{2} \mathrm{SiO}_{3}\right)$ líquido com módulo de sílica $\left(\mathrm{Ms}=\mathrm{SiO}_{2} / \mathrm{Na}_{2} \mathrm{O}\right)$ de 2,23. O agregado miúdo utilizado foi a areia de quartzo (sílica cristalina).

Tabela 1 - Composição do Metacaulim

\begin{tabular}{l|c|c|c|c|c|c}
\hline Composição (\%) & $\mathrm{SiO}_{2}$ & $\mathrm{Al}_{2} \mathrm{O}_{3}$ & $\mathrm{Fe}_{2} \mathrm{O}_{3}$ & $\mathrm{Na}_{2} \mathrm{O}$ & $\mathrm{K}_{2} \mathrm{O}$ & $\mathrm{PF}$ \\
\hline Metacaulim & 51,54 & 40,50 & 2,80 & 0,08 & 0,18 & 2,62 \\
\hline
\end{tabular}

A Figura 1A mostra que os tamanhos das partículas do metacaulim variam de tamanhos da cerca de $0,5 \mu \mathrm{m}$, até valores um pouco maiores que $100 \mu \mathrm{m}$, contudo a maior concentração se encontra entre 10 e $100 \mu \mathrm{m}$. O ensaio de tempo de ajuste da pasta geopolimérica (Figura 1B) foi feito em um Vicat eletrônico, onde a pasta foi colocada em um molde cilíndrico de 40 $\mathrm{mm}$ de altura, com o início da contagem do tempo ao primeiro contato dos materiais na mistura, e foi medida a profundidade da perfuração da agulha a cada 2 minutos. Foi determinado o início do ajuste quando a agulha marcou o primeiro furo de profundidade igual ou imediatamente superior a $36 \mathrm{~mm}$, e o fim de pega quando a agulha atingiu a profundidade mínima, ou seja, não penetrou mais a amostra.

A

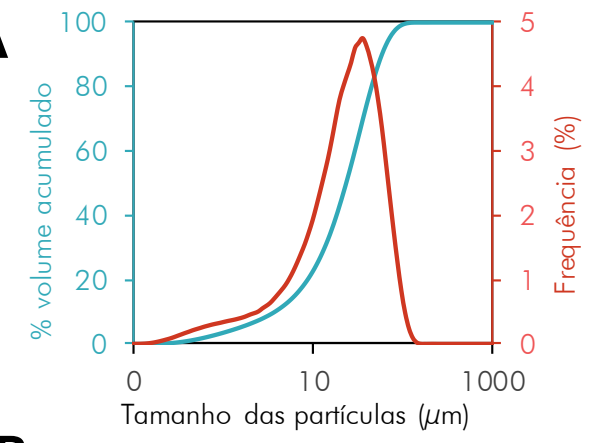

B

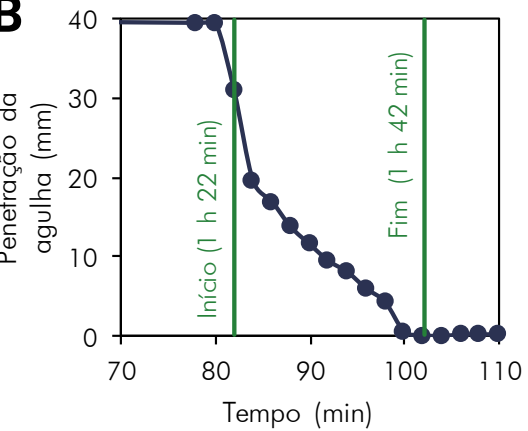

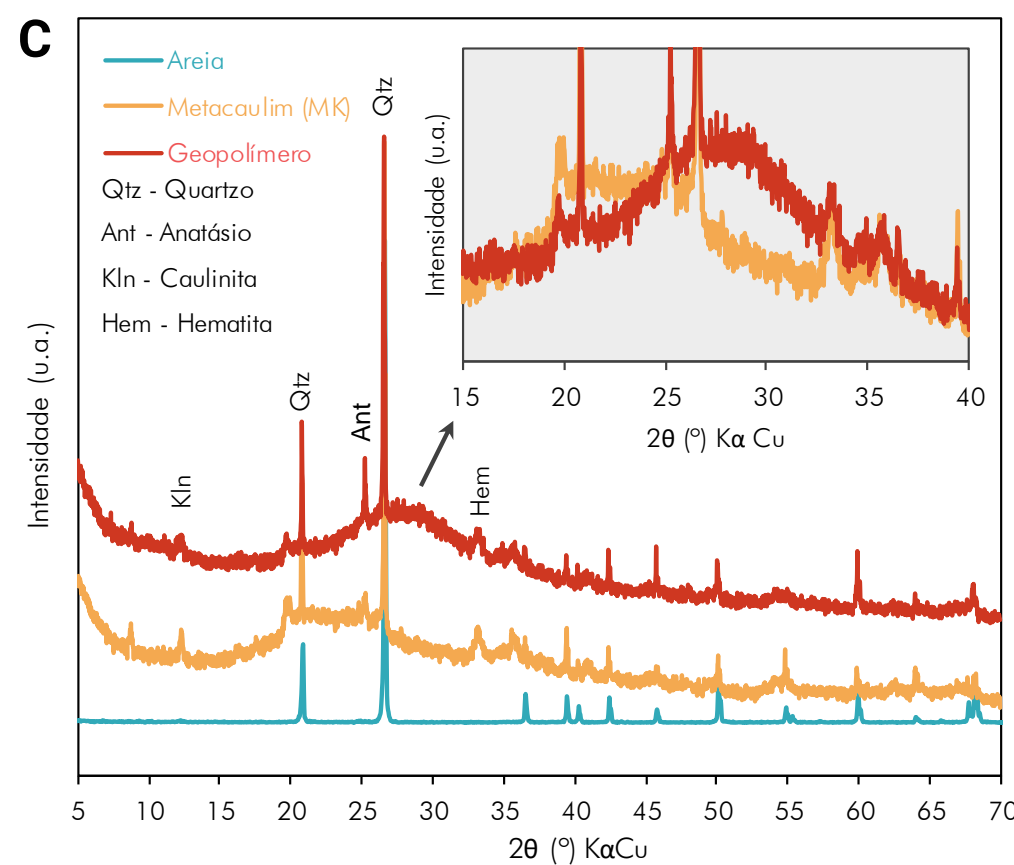

Figura 1: Caracterização dos materiais. Em (A) granulometria a laser do metacaulim, (B) tempo de início e fim de ajuste da pasta geopolimérica, (C) difratogramas da areia, do metacaulim e do geopolímero ativado alcalinamente.

A Difração de Raio X (DRX) da areia (Figura 1C) identificou os picos referentes ao quartzo cristalino, mostrando a composição esperada. As difrações do metacaulim e do geopolímero ativado alcalinamente mostraram que as fases 
cristalinas se mantiveram mesmo após a ativação por não participarem da ativação alcalina, porém é possível ver uma variação considerável entre o metacaulim e o geopolímero, entre $18^{\circ}$ e $25^{\circ}$ a intensidade relativamente menor do geopolímero indica que o material semicristalino e amorfo do metacaulim se tornou quase completamente amorfo (WANG; LI; YAN, 2005).

\subsection{Métodos}

\subsubsection{Beneficiamento dos agregados}

Primeiramente a areia foi lavada para remoção de impurezas que pudessem afetar os resultados do estudo, a secagem foi ao ar livre, então foi peneirada e as partículas divididas nas faixas granulométricas 1,20-0,60 mm;0,60-0,30 mm; 0,30-0,15 $\mathrm{mm}$. O primeiro número indica a malha da peneira em milímetros $(\mathrm{mm})$ em que as partículas foram passantes, e o segundo número indica a abertura das peneiras em milímetros em que as partículas foram retidas, indicando assim, uma faixa de tamanho das partículas de agregado entre as duas malhas. Ao longo do trabalho, as famílias de cada faixa granulométrica serão nomeadas de acordo com a Tabela 2, a nomenclatura adotada somente indica a malha da peneira em que as partículas de agregado foram retidas, em $\mu \mathrm{m}$. A divisão em frações foi feita para moldar as argamassas com tamanhos diferentes das partículas de agregados.

Tabela 2 - Definição das faixas granulométricas do agregado

\begin{tabular}{c|c|c}
\hline Nomenclatura adotada para as amostras & Peneira Passante $(\mathbf{m m})$ & Peneira Retida (mm) \\
\hline $\mathbf{6 0 0}$ & 1,20 & 0,60 \\
\hline $\mathbf{3 0 0}$ & 0,60 & 0,30 \\
\hline $\mathbf{1 5 0}$ & 0,30 & 0,15 \\
\hline
\end{tabular}

\subsubsection{Preparo das argamassas}

Inicialmente, foram homogeneizados o precursor (metacaulim), o hidróxido de sódio $(\mathrm{NaOH})$ em escamas, e a areia de quartzo divididas nas 3 famílias de faixas granulométricas, depois foram acrescentados o silicato de sódio $\left(\mathrm{Na}_{2} \mathrm{SiO}_{3}\right)$ e a água, na mesma quantidade independente dos tamanhos das partículas de areia para que a quantidade de água não interfira na variação dos resultados. As argamassas foram misturadas mecanicamente por um período de cinco minutos em um misturador mecânico, contados a partir da adição da água na mistura. A Tabela 3 mostra o consumo dos materiais.

Tabela 3 - Consumo dos materiais na mistura

\begin{tabular}{c|c|c|c|c}
\hline \multicolumn{5}{|c|}{ Consumo em Kg/m } \\
\hline MK & Areia & $\mathrm{NaOH}$ & $\mathrm{Na}_{2} \mathrm{SiO}_{3}$ & $\mathrm{H}_{2} \mathrm{O}$ \\
\hline 458,47 & 916,94 & 36,68 & 302,59 & 229,24 \\
\hline
\end{tabular}

\subsubsection{Espalhamento no mini-slump}

O ensaio no mini-slump foi realizado para medir a consistência das argamassas, medindo o diâmetro de espalhamento das amostras logo após o término do processo de mistura dos materiais.

\subsubsection{Retração e perda de massa}

Após a moldagem o ensaio seguiu os procedimentos da NBR 15261 (ABNT, 2005), com adaptações na cura das amostras e nas idades de medições. Após a moldagem, as amostras foram embrulhadas em plástico para evitar a perda de água durante o endurecimento do gel geopolimérico, e foram mantidos na câmara térmica por 24 horas, a $25^{\circ} \mathrm{C}$ e $50 \%$ de umidade, então desmoldados para a primeira medida no relógio comparador com $24 \mathrm{~h}$ após a moldagem e mantidos na câmara durante 28 dias, as medidas foram feitas nas idades de 7, 14, 21 e 28 dias. Concomitante ao ensaio de retração, foi feito a medição da perda de massa, pesando as amostras nas mesmas idades em que foram medidas para retração.

\subsubsection{Absorção total de água, índice de vazios e massa específica aparente}

As amostras utilizadas para absorção foram serradas dos corpos-de-prova do ensaio de retração após os 28 dias de ensaio, o procedimento seguiu a norma ASTM C642 - 13, e os resultados serviram para calcular a absorção, o índice de vazios e a massa especifica aparente das argamassas. 


\subsubsection{Compressão axial}

Para o ensaio de compressão axial das argamassas, foram utilizados os moldes cilíndricos de $5 \mathrm{~cm}$ de diâmetro e $10 \mathrm{~cm}$ de altura, e foram colocados em cura controlada na câmara térmica a $25^{\circ} \mathrm{C}$ de temperatura e $50 \%$ de umidade, a cura durou 28 dias. Após o término da cura as amostras foram ensaiadas a compressão seguindo a NBR 7215 (ABNT, 2019).

\subsubsection{Análise dos dados}

Os dados foram analisados quanto a sua normalidade e homocedasticidade, quando os dados apresentavam distribuição normal e homogeneidade das variâncias, estes foram analisados utilizando-se análise de variância de uma via (ANOVA de uma via) por conter mais de duas condições experimentais, caso contrário, os dados foram analisados utilizando-se ANOVA de Kruskal-Wallis, com fator de correção de Bonferroni. Para avaliar a correlação entre a perda de massa do material com a retração linear, foi utilizado regressão linear simples. Foram consideradas diferenças significativas quando $\mathrm{P}<0,05$.

\section{RESULTADOS E DISCUSSÃO}

\subsection{Espalhamento no mini-slump}

Os resultados do ensaio de mini-slump mostraram que o tamanho das partículas de agregado interferiram na reologia das argamassas, quanto ao diâmetro de espalhamento, a argamassa com os menores tamanhos de agregados apresentaram o menor diâmetro de espalhamento (Figura 1A) devido a uma maior superfície específica das partículas menores, deixando menos água livre para a pasta. Enquanto as argamassas de 300 e 600 não apresentaram diferenças no diâmetro de espalhamento, é possível identificar uma quantidade maior de água livre na argamassa de 600 (Figura 1B).

Esse resultado é importante para inferir sua trabalhabilidade, pois ajuda a determinar a facilidade que essa argamassa pode ser misturada, aplicada e consolidada de forma homogênea (KOEHLER, 2009), ou seja, medindo a facilidade que o material tem de preencher vazios nos moldes. Na prática isso significa evitar ou diminuir o surgimento de vazios de moldagens ou falta de homogeneidade na mistura controlando o tamanho dos agregados, pois para cada necessidade pode ser adotado uma granulometria diferente para o agregado. Outros fatores como o ângulo de atrito e densidade aparente do agregado seco também podem influenciar na consistência das argamassas (HU; WANG, 2011), porém essas variáveis não foram tratadas neste estudo.
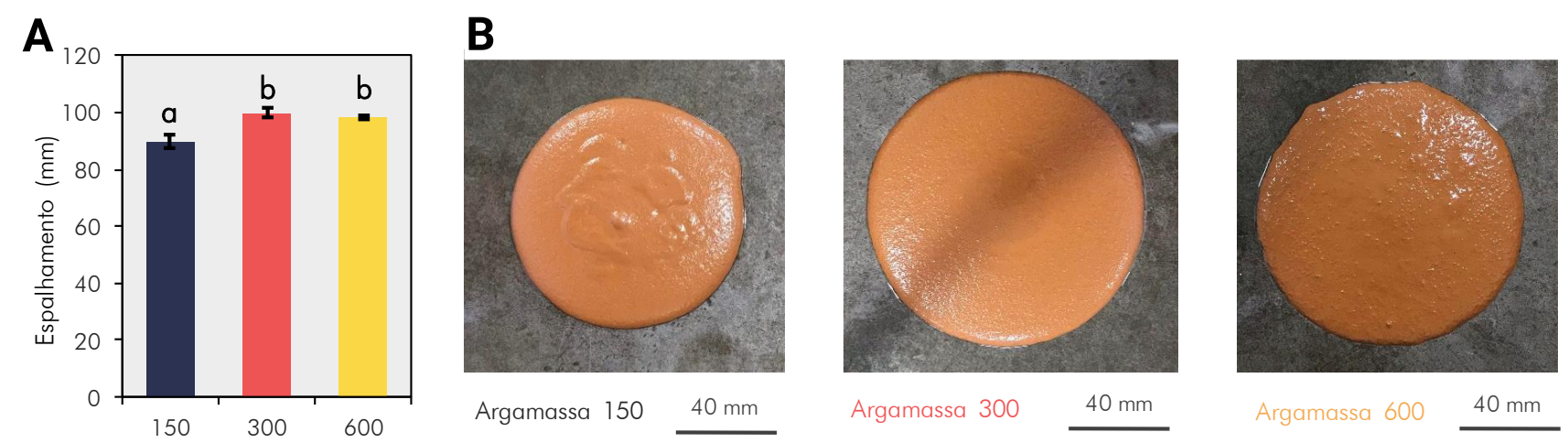

Figura 1 - Espalhamento. (A) os resultados para os diâmetros de espalhamento das amostras, (B) fotografias das argamassas espalhadas. Em (A) Valores expressados em médias \pm desvio padrão. Letras minúsculas distintas indicam diferença estatística quando comparado entre as argamassas para a mesma variável (ANOVA de uma via; $\mathrm{P}<0,05$ )

\subsection{Absorção total de água, índice de vazios e massa específica aparente}

O resultado de absorção total de água (Figura 2A) indicou que a absorção se mostrou semelhante nas amostras de 150 e 600, porém a de 300 apresentou uma absorção maior se comparada com a maior granulometria (600), enquanto o índice de vazios não apresentou variação significativa com o aumento do tamanho do agregado (Figura 2B), explicado devido ao tamanho dos poros gerados na argamassa, pois os geopolímeros costumam apresentar poros menores, porém em maior quantidade (HAJIMOHAMMADI; PROVIS; VAN DEVENTER, 2011), indicando que a porcentagem de água absorvida pode ser menor devido a não conseguir adentrar nos poros menores, porém o índice de vazios permanece constante, indicando que não há diferença no volume total dos poros conectados. 
A

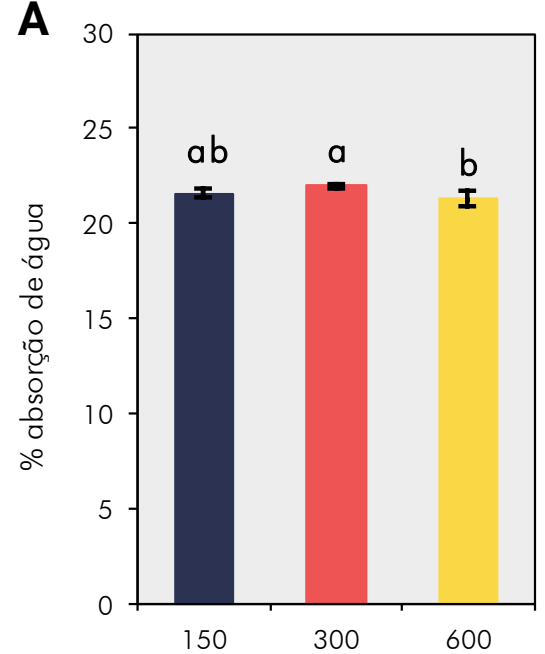

B

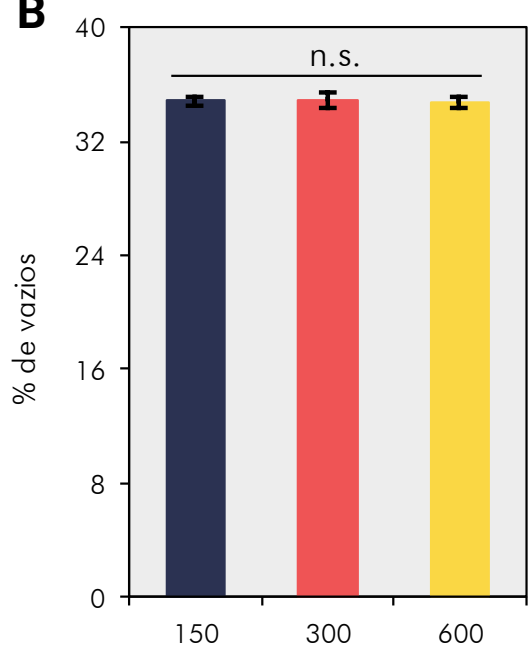

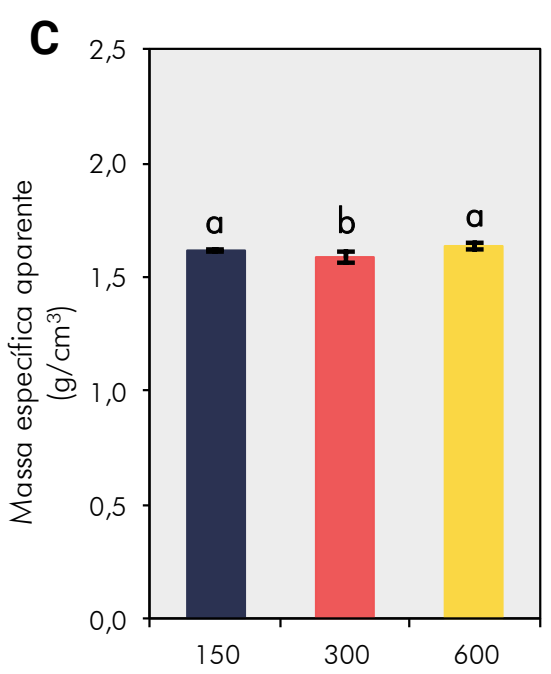

Figura 2: Propriedades físicas das argamassas. (A) maiores partículas implicam em menores valores médios de absorção de água, (B) mas não influenciam no índice de vazios. (C) Massa específica aparente das argamassas. Valores expressados em médias \pm desvio padrão, o número de repetições é 6 . Letras minúsculas distintas indicam diferença estatística quando comparado entre as argamassas para a mesma variável (ANOVA de uma via; $\mathrm{P}<0,05$ ), n.s. é não significativo.

A explanação das amostras com agregados de maior tamanho (600) possuírem uma menor absorção quando comparados com as amostras de tamanho intermediário (300) (Figura 2A) pode ser explicado pelo empacotamento dos grãos, pois partículas com maior variação de tamanhos de agregado tendem a aumentar o empacotamento do material, e isso acontece para as amostras de 600, por ter uma maior variação do tamanho das partículas pois apresentam partículas entre $600 \mu \mathrm{m}$ e $1200 \mu \mathrm{m}$, ou seja uma variação de $600 \mu \mathrm{m}$, enquanto as amostras de 300 apresentam uma dispersão que varia somente de $300 \mu \mathrm{m}$ a $600 \mu \mathrm{m}$ em suas partículas. Enquanto as amostras de 150 e 600 não apresentaram diferença significativa nos dados de absorção de água (Figura 2A), indicando que partículas menores de agregado também causam um empacotamento menor e consequentemente uma diminuição da absorção.

De forma geral, esses resultados indicam que a diminuição da variação do tamanho das partículas de agregado de 600 para 300 indicam maior quantidade de poros penetrados por água, porém de 300 para 150 essa variação foi insignificativa devido a diminuição das partículas causar um aumento do empacotamento. Essa ideia também é suportada pelos dados de massa específica aparente (Figura 2C), que indicam uma densidade aparente menor para as amostras de 300, como esse valor de massa considera os vazios, um valor menor significa maior quantidade de vazios, indicando que as amostras de 300 realmente apresentam um menor empacotamento quando comparadas com as amostras que apresentam uma variação maior nos tamanhos das partículas (600) e as amostras que apresentam partículas menores (150).

\subsection{Retração e perda de massa}

A perda de massa teve um aumento na idade mais precoce (7 dias), nas datas posteriores não apresentou mudanças, provavelmente indicando a perda de água intersticial não ligada quimicamente que costuma acontecer nos primeiros dias em geopolímeros, ou seja, a retração por secagem (YANG; ZHU; ZHANG, 2017). Nas demais medições a perda de massa se mostrou constante ao longo do tempo para todas as famílias, provavelmente por causa da retração autógena que ocorre a partir do primeiro dia e dura enquanto o material estiver ativo quimicamente devido reorganização e geopolimerização da estrutura de aluminossilicatos (MA; YE, 2015), e não apresenta perda de massa significativa (ZHANG et al., 2012; MA; YE, 2015). (Figura 3A).

Enquanto as amostras de 150 e 300 mostraram resultados semelhantes, a perda de massa em 600 foi maior em todas as idades, isso pode ser explicado baseando-se nos resultados de absorção de água e densidade aparente (Figura 2A e 2C), onde indicam que a argamassa de 600 apresentam, além um volume menor de poros, provavelmente poros de tamanhos menores. Poros com diâmetros menores causam um aumento nas tensões capilares (MA; YE, 2015) e consequentemente um aumento na perda de água (YANG; ZHU; ZHANG, 2017). 
Em relação à retração linear das argamassas, o aumento da idade implica em maiores respostas à retração, devido a provável retração por secagem e a autógena, a primeira até os 7 dias e a segunda ao longo de todo ensaio, e quanto maior o tamanho das partículas, maior a taxa de retração linear com o aumento da idade (Figura 3B), esses resultados indicam que o tamanho das partículas de agregado, como nas argamassas a base de cimento Portland, também influencia nas retração de argamassas geopoliméricas. Contudo, em concretos e argamassas de cimento Portland, o aumento da área específica dos agregados gera maiores retrações (ZHANG; ZAKARIA; HAMA, 2013), enquanto nossos resultados mostram justamente o contrário, visto que as partículas menores de agregado, que consequentemente tendem a apresentar maiores áreas especificas, apresentaram menores variações dimensionais causadas pela retração.

Os resultados sugerem que não há correlação entre essas variáveis para todas as variações de partículas, esses resultados representam a existência de duas causas distintas para a perda de massa e a retração, provavelmente a retração por secagem e a retração autógena respectivamente. A retração autógena representa uma parcela muito pequena da perda de massa, e causa retração ao longo da geopolimerização, explicando os resultados de retração. Enquanto a retração por secagem ocorre antes dos 7 dias e é a grande responsável pela perda de massa do material devido, explicando os resultados de perda de massa (YANG; ZHU; ZHANG, 2017).

Foi combinado o conjunto de dados da taxa de perda de massa das argamassas com as respostas à retração, e apresentaram um $\mathrm{r}^{2}$ razoavelmente baixo (Figura 3C-E), indicando que a retração não é correlacionada com a perda de massa na argamassa geopolimérica. A perda de massa foi significativa somente até os 7 dias, nas datas posteriores não apresentou mudanças, indicando a perda de água característica da retração por secagem, pois é durante a saída da água livre que acontece a perda mais significativa de massa, e essa saída tende a ser nos dias inicias pois a água não participa da reação de geopolimerização (ZUHUA et al., 2009). A retração apresentou um acréscimo maior aos 7 dias devido a retração por secagem, porém continuou aumentando até a última medição aos 28 dias, indicando que após os 7 dias quando a perda de água cessou, a retração autógena, ainda está acontecendo, causando assim uma retração que continua mesmo sem a perda de água.

\section{A}

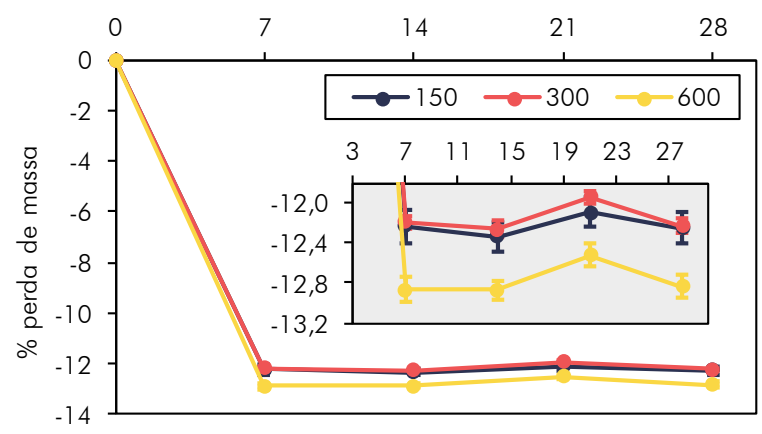

B

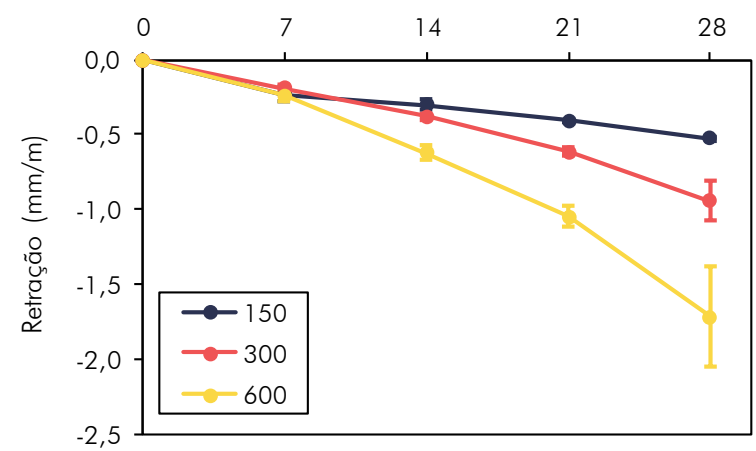

E

$\%$ perda de massa
C $\%$ perda de massa

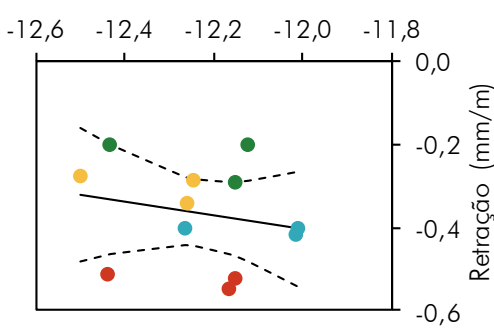

D
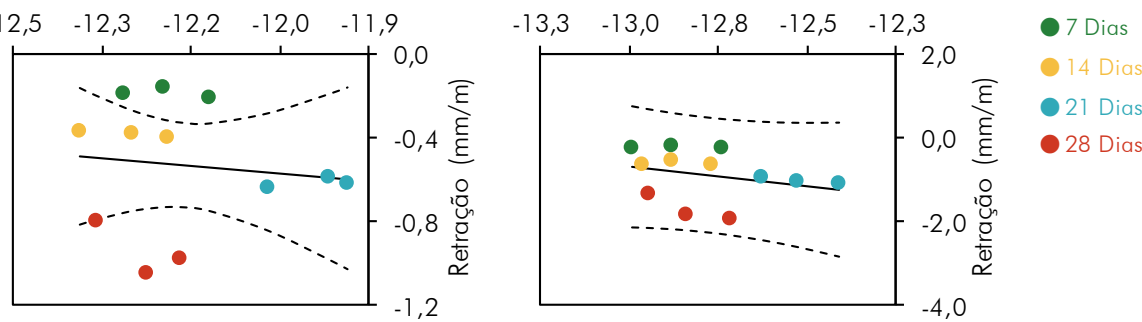

Figura 3: Variações de massa e dimensionais das argamassas. Em (A) taxa de perda de massa, em (B) retração linear das argamassas, em ambos foram utilizadas ANOVA de Kruskal-Wallis para comparar os efeitos da idade e do tamanho das partículas. A regressão linear simples é apresentada de (C-E), sendo (C) para as argamassas com partículas de tamanho 150 $\mu \mathrm{m}\left(\mathrm{r}^{2}=0,051 ; \mathrm{P}=0,477\right)$, em (D) para $300 \mu \mathrm{m}\left(\mathrm{r}^{2}=0,013 ; \mathrm{P}=0,718\right)$ e em $(\mathrm{E})$ para $600 \mu \mathrm{m}\left(\mathrm{r}^{2}=0,079 ; \mathrm{P}=0,373\right)$. Em (A) e (B) os valores são expressos em médias \pm desvio padrão e de (C-E) os pontos representam os dados brutos, linhas tracejadas são intervalos de confiança para $95 \%$.

Um fator determinante na retração de compósitos, principalmente os ligantes utilizados na construção civil, é a: zona de transição entre a matriz e os agregados (MARUYAMA; SUGIE, 2014), devido justamente a composição porosa da zona de transição, que possui baixo módulo de elasticidade e uma facilidade de retrair bem maior que a matriz e o agregado nos 
materiais a base de cimento Portland. Nos geopolímeros, estudos recentes apontam que a zona de transição desses materiais é fortalecida pela composição do agregado, no caso, os agregados silicosos, como o de quartzo utilizado neste estudo (WAN et al., 2017; RIAHI et al., 2020). A dissolução superficial do silício presente nesses agregados durante a síntese no ambiente alcalino e a presença de um silicato amorfo na mistura, gera um aumento de resistência na zona de transição (WAN et al., 2017; RIAHI et al., 2020), pois a relação Silício/Alumínio aumenta, e ela é a principal responsável pela resistência de materiais geopoliméricos (DUXSON et al., 2007b; HE et al., 2016), porém não afeta a resistência geral da matriz pois a dissolução é somente superficial.

Esse resultado mostra que diferentemente dos materiais a base de cimento Portland convencional, os materiais geopoliméricos apresentam um ganho de resistência aos esforços internos de retração quanto menores as partículas, devido a sua zona de transição apresentar composição e propriedades semelhantes a própria matriz (LEE; VAN DEVENTER, 2004; PACHECO-TORGAL; CASTRO-GOMES; JALALI, 2007; DEMIE; NURUDDIN; SHAFIQ, 2013), indicando uma zona de transição fortalecida.

Sendo assim, se tratando do uso de agregados de quartzo, a porosidade da zona de transição que é a principal causa de retração em argamassas de cimento Portland convencional, não causam esse efeito em argamassas geopoliméricas, devido a um provável fortalecimento interno do material com as zonas ao redor dos agregados com maior quantidades de silício, explicando o porquê da retração diminuir com a diminuição do tamanho das partículas de agregado, pois quanto menores as partículas, uma maior quantidade de grãos estará presente na argamassa para uma mesma massa, equivalendo a uma maior quantidade de pontos de fortalecimento interno contra os esforços de retração, assim como partículas menores de agregado tendem a possuir maior superfície específica, aumentando a superfície dissolvida, auxiliando na durabilidade do material.

\subsection{Resistencia à compressão}

O elo mais fraco em compósitos é conhecidamente a zona de transição, por geralmente não apresentar propriedades tão altas quanto os demais componentes do material, principalmente no caso dos materiais a base de cimento Portland convencional (ULRIK NILSEN; MONTEIRO, 1993; AKÇAOĞLU; TOKYAY; ÇELIK, 2005). Foi analisado as respostas à compressão das argamassas variando o tamanho das partículas de agregado. Não houve diferença significativa na resistência à compressão (Figura 4), mesmo para a família com o agregado de maior tamanho das partículas, o que também implica em dizer que o aumento da zona de transição não alterou a resistência. Os resultados anteriores sugerem fortemente que na argamassa geopolimérica com agregados de quartzo, a dissolução superficial do silício no meio alcalino fortalece a zona de transição, entretanto, não influencia na matriz (RIAHI et al., 2020), assim, era esperado que a resistência permanecesse semelhante para todas a amostras, pois a zona de transição não se apresenta como o elo mais fraco do compósito.

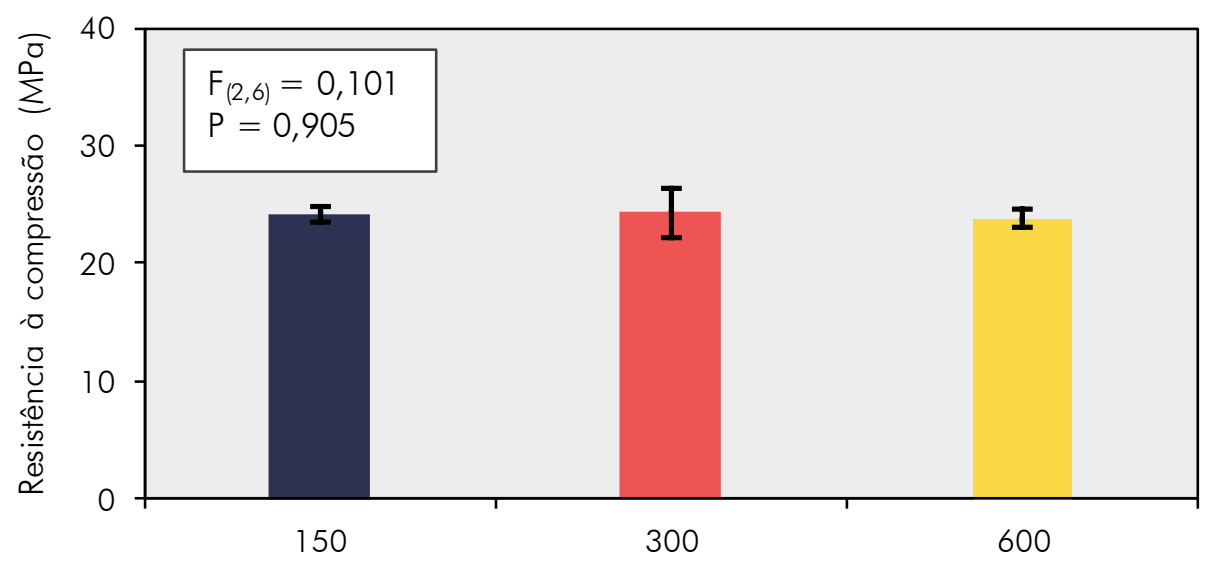

Figura 4: Resposta à compressão das argamassas geopoliméricas. O aumento do tamanho das partículas não influencia na resistência à compressão. Valores expressados em médias \pm desvio padrão, o número de repetições é 3. (ANOVA de uma via). 


\subsection{Perspectivas}

Estudos de durabilidade em um material novo, são promissores, e tem um significado muito grande para o combate as patologias, pois conhecendo as propriedades de durabilidade do material podemos melhor entender seu comportamento sob o ataque de agentes agressivos e como recuperar esses materiais. Além disso os resultados implicam em impactos econômicos e ambientais para o geopolímero, visto que o fato da durabilidade prática dos geopolímeros serem desconhecidas devido ser um material novo, ser uma das principais barreiras para uma competitividade alta entre os ligantes no mercado (VAN DEVENTER; PROVIS; DUXSON, 2012), e o aumento da durabilidade de construções e infraestruturas caracterizar uma menor variação de estoques de materiais (MÜLLER et al., 2013), gerando um uso menor de matéria prima para reparar ou refazer estruturas, reduzindo o uso de energia e as emissões de gases de efeito estufa.

\section{CONCLUSÕES}

Os resultados mostram-se promissores para o entendimento da durabilidade da argamassa geopolimérica e consequentemente no combate as patologias. A consistência por exemplo, mostrou que a diminuição do tamanho das partículas, causa uma diminuição do espalhamento, provavelmente por causa da diminuição da água livre com o aumento da superfície específica do agregado. Resultado importante para controlar a trabalhabilidade do material, pois com o controle do tamanho dos agregados pode-se controlar sua consistência, evitando o surgimento de vazios de moldagens ou a falta de homogeneidade na mistura, pois diminuem a resistência e a durabilidade do material, facilitando a entrada de agente agressivos e o surgimento de patologias.

A absorção de água, índice de vazios e densidade aparente mostraram uma relação com a variação de tamanho dos agregados, indicando que essas propriedades são influenciadas pelo empacotamento que a variação das faixas granulométricas dos agregados provoca. Isso indica que o controle do empacotamento do material é de extrema importância para a durabilidade de argamassas geopoliméricas, em vista que diminuir a absorção e os vazios, também diminui a permeabilidade do material, diminuindo o aparecimento de patologias.

A retração e a perda de massa não apresentaram correlação. A perda de massa é causada pela retração por secagem, enquanto a variação dimensional foi causada principalmente pela retração autógena, mas também pela retração por secagem. E também as partículas de agregado menores geram retrações menores nas argamassas geopoliméricas, devido a maior dissolução do silício na zona de transição aumentando sua resistência aos esforções internos, causadores da variação dimensional. De forma geral, o controle da retração pode ser feito de maneira efetiva somente controlando o tamanho dos agregados, descoberta muito importante para entender suas propriedades e prever seus comportamentos, como surgimento de fissuras provenientes da variação dimensional ou perda excessiva de água.

A resistência a compressão não apresentou correlação com a variação do tamanho do agregado, isso indica que a zona de transição não apresenta diferença significativa em resistência quando comparada com a matriz, pois com a variação das superfícies especificas dos agregados, houve variação no tamanho da zona de transição, e essa última não influenciou na resistência a compressão.

Esse trabalho concluiu que o tamanho das partículas interfere na durabilidade, mas de maneiras diferentes dependendo da propriedade analisada, mas de forma geral o empacotamento dos grãos, o tipo de agregado, e a área especifica dos mesmos, são os principais responsáveis pela interferência na durabilidade. As implicações desses resultados, além de colaborar com a teoria do fortalecimento da zona de transição, também implica no controle da durabilidade desses materiais através da escolha do tamanho das partículas do agregado. Estudos de durabilidade em um material novo, são promissores, e tem um significado muito grande para o combate as patologias.

\section{AGRADECIMENTOS}

Os autores agradecem: ao Laboratório de Materiais Cerâmicos (LACER) da UFRGS, pela granulometria a laser e FRX do metacaulim; ao laboratório de Raios X, analise Térmica e Dielétrica do PPGF da UFPA pela análise de DRX do metacaulim; a CAPES e a PROPESP-UFPA pelo auxílio financeiro em forma de bolsa de iniciação cientifica e de pósgraduação; e também ao grupo Avanços na Ciência do Geopolímero (ACGEO), pelos debates e contribuições dos seus integrantes. 


\section{REFERÊNCIAS}

AKÇAOĞLU, T.; TOKYAY, M.; ÇELIK, T. Assessing the ITZ microcracking via scanning electron microscope and its effect on the failure behavior of concrete. Cement and Concrete Research, 2005.

BOIVIN, S. et al. Experimental assessment of chemical shrinkage of hydrating cement paste. In: In: Tazawa EI, editor. Proceedings of the international workshop on autogenous shrinkage of concrete (Autoshrink'98), Anais...1999.

BRUNE, P. F. et al. The fracture toughness of an Imperial Roman mortar. Engineering Fracture Mechanics, v. 102, p. 65-76, 2013. Disponível em: 〈http://dx.doi.org/10.1016/j.engfracmech.2013.02.007>.

CHEAH, C. B.; RAMLI, M. Mechanical strength, durability and drying shrinkage of structural mortar containing HCWA as partial replacement of cement. Construction and Building Materials, 2012.

CHOI, M. S. et al. Effect of the coarse aggregate size on pipe flow of pumped concrete. Construction and Building Materials, 2014.

DELATTE, N. J. LESSONS FROM ROMAN CEMENT AND CONCRETE. Journal of Professional Issues in Engineering Education and Practice, v. 127, n. July, p. 109-115, 2001.

DEMIE, S.; NURUDDIN, M. F.; SHAFIQ, N. Effects of micro-structure characteristics of interfacial transition zone on the compressive strength of self-compacting geopolymer concrete. Construction and Building Materials, 2013.

DUXSON, P. et al. Geopolymer technology: The current state of the art. Journal of Materials Science, v. 42, n. 9, p. 2917-2933, 2007a.

DUXSON, P. et al. The effect of alkali and Si/Al ratio on the development of mechanical properties of metakaolin-based geopolymers. Colloids and Surfaces A: Physicochemical and Engineering Aspects, 2007b.

HAJIMOHAMMADI, A.; NGO, T.; VONGSVIVUT, J. Interfacial chemistry of a fly ash geopolymer and aggregates.

Journal of Cleaner Production, v. 231, p. 980-989, 2019. Disponível em:
<https://doi.org/10.1016/j.jclepro.2019.05.249>.

HAJIMOHAMMADI, A.; PROVIS, J. L.; VAN DEVENTER, J. S. J. The effect of silica availability on the mechanism of geopolymerisation. Cement and Concrete Research, 2011.

HANSEN, W. Drying Shrinkage Mechanisms in Portland Cement Paste. Journal of the American Ceramic Society, 1987.

$\mathrm{HE}$, P. et al. Effects of Si/Al ratio on the structure and properties of metakaolin based geopolymer. Ceramics International, v. 42, n. 13, p. 14416-14422, 2016. Disponível em: 〈http://dx.doi.org/10.1016/j.ceramint.2016.06.033>.

HU, J.; WANG, K. Effect of coarse aggregate characteristics on concrete rheology. Construction and Building Materials, 2011.

JACKSON, M. D. et al. Mechanical resilience and cementitious processes in Imperial Roman architectural mortar. Proceedings of the National Academy of Sciences of the United States of America, 2014.

JACKSON, M. D. et al. Phillipsite and Al-tobermorite mineral cements produced through low-temperature water-rock reactions in Roman marine concrete. American Mineralogist, 2017.

KLYSZ, G.; BALAYSSAC, J. P. Determination of volumetric water content of concrete using ground-penetrating radar. Cement and Concrete Research, 2007.

KOEHLER, E. P. Use Rheology to specify, Design and manage self-consolidating concrete. In: 9th ACI International Conference on Superplasticizers \& 10th International Conference on Recent, Seville, Spain. Anais... Seville, Spain: 2009.

KUENZEL, C. et al. Ambient temperature drying shrinkage and cracking in metakaolin-based geopolymers. Journal of 
the American Ceramic Society, 2012.

LEE, W. K. W.; VAN DEVENTER, J. S. J. The interface between natural siliceous aggregates and geopolymers. Cement and Concrete Research, v. 34, n. 2, p. 195-206, 2004.

MA, Y.; YE, G. The shrinkage of alkali activated fly ash. Cement and Concrete Research, 2015.

MARUYAMA, I.; SUGIE, A. Numerical study on drying shrinkage of concrete affected by aggregate size. Journal of Advanced Concrete Technology, 2014.

MERMERDAŞ, K. et al. Effect of aggregate properties on the mechanical and absorption characteristics of geopolymer mortar. Engineering Science and Technology, an International Journal, v. 20, n. 6, p. 1642-1652, 2017.

MÜLLER, D. B. et al. Carbon emissions of infrastructure development. Environmental Science and Technology, 2013.

PACHECO-TORGAL, F.; CASTRO-GOMES, J.; JALALI, S. Investigations about the effect of aggregates on strength and microstructure of geopolymeric mine waste mud binders. Cement and Concrete Research, 2007.

PERERA, D. S. et al. Influence of curing schedule on the integrity of geopolymers. Journal of Materials Science, 2007.

PLINIO, G. Naturalis Historiae. [s.l: s.n.]

RIAHI, S. et al. The effect of mixing molar ratios and sand particles on microstructure and mechanical properties of metakaolin-based geopolymers. Materials Chemistry and Physics, 2020.

SCHIESSL, P. Durability of reinforced concrete structures. Construction and Building Materials, v. 10, n. 5, p. 289292, 1996.

SHIN, H. C.; LANGE, D. A. Effects of shrinkage and temperature in bonded concrete overlays. ACI Materials Journal, 2004.

STEINEROVA, M. Mechanical properties of geopolymer mortars in relation to their porous structure. Ceramics - Silikaty, v. 55, n. 4, p. 362-372, 2011.

ULRIK NILSEN, A.; MONTEIRO, P. J. M. Concrete: A three phase material. Cement and Concrete Research, 1993.

VAN DEVENTER, J. S. J.; PROVIS, J. L.; DUXSON, P. Technical and commercial progress in the adoption of geopolymer cement. Minerals Engineering, 2012.

WAN, Q. et al. Combination formation in the reinforcement of metakaolin geopolymers with quartz sand. Cement and Concrete Composites, v. 80, p. 115-122, jul. $2017 . \quad$ Disponível em: <https://linkinghub.elsevier.com/retrieve/pii/S0958946516303080>.

WANG, H.; LI, H.; YAN, F. Synthesis and mechanical properties of metakaolinite-based geopolymer. Colloids and Surfaces A: Physicochemical and Engineering Aspects, 2005.

YANG, T.; ZHU, H.; ZHANG, Z. Influence of fly ash on the pore structure and shrinkage characteristics of metakaolinbased geopolymer pastes and mortars. Construction and Building Materials, v. 153, p. 284-293, out. 2017. Disponível em: <https://linkinghub.elsevier.com/retrieve/pii/S0950061817309595>.

ZHANG, W.; ZAKARIA, M.; HAMA, Y. Influence of aggregate materials characteristics on the drying shrinkage properties of mortar and concrete. Construction and Building Materials, 2013.

ZHANG, Z. et al. Quantitative kinetic and structural analysis of geopolymers. Part 1. the activation of metakaolin with sodium hydroxide. Thermochimica Acta, 2012.

ZHUANG, X. Y. et al. Fly ash-based geopolymer: Clean production, properties and applications. Journal of Cleaner 


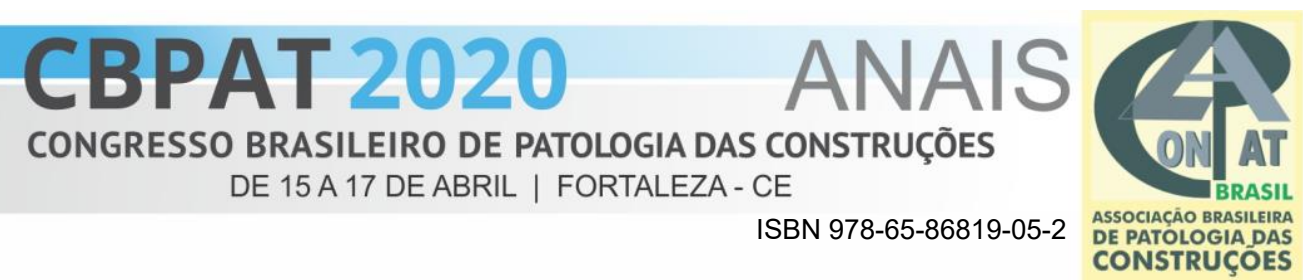

Production, v. 125, p. 253-267, 2016. Disponível em: 〈http://dx.doi.org/10.1016/j.jclepro.2016.03.019>.

ZUHUA, Z. et al. Role of water in the synthesis of calcined kaolin-based geopolymer. Applied Clay Science, v. 43, n. 2, p. 218-223, 2009. Disponível em: 〈http://dx.doi.org/10.1016/j.clay.2008.09.003〉. 